\title{
THE IMPACT OF THE GAS DISTRIBUTION ON THE DETERMINATION OF DYNAMICAL MASSES OF GALAXIES USING UNRESOLVED OBSERVATIONS
}

\author{
W. J. G. DE BLOK ${ }^{1,2,3}$ AND FABIAN WALTER ${ }^{4}$ \\ ${ }^{1}$ Netherlands Institute for Radio Astronomy (ASTRON), Postbus 2, 7990 AA Dwingeloo, The Netherlands \\ 2 Astrophysics, Cosmology and Gravity Centre, University of Cape Town, Private Bag X3, Rondebosch 7701, South Africa \\ ${ }^{3}$ Kapteyn Astronomical Institute, University of Groningen, P.O. Box 800, 9700 AV Groningen, The Netherlands \\ ${ }^{4}$ Max-Planck Institut für Astronomie, Königstuhl 17, D-69117, Heidelberg, Germany \\ Received 2013 October 18; accepted 2014 January 30; published 2014 March 24
}

\begin{abstract}
Dynamical mass $\left(M_{\mathrm{dyn}}\right)$ is a key property of any galaxy, yet a determination of $M_{\mathrm{dyn}}$ is not straightforward if spatially resolved measurements are not available. This situation occurs in single-dish H I observations of the local universe, but also frequently in high-redshift observations. $M_{\mathrm{dyn}}$ measurements in high-redshift galaxies are commonly obtained through observations of the $\mathrm{CO}$ line, the most abundant tracer of the molecular medium. Even though in most cases the $\mathrm{CO}$ line width can be determined with reasonable accuracy, a measurement of the size of the emitting region is typically challenging given current facilities. We show how the integrated spectra ("global profiles") of a variety of galaxy models depend on the spatial distribution of the tracer gas as well as its velocity dispersion. We demonstrate that the choice of tracer emission line (e.g., H I tracing extended, "flat," emission versus CO tracing more compact, "exponential," emission) significantly affects the shape of the global profiles. In particular, in the case of high $\left(\sim 50 \mathrm{~km} \mathrm{~s}^{-1}\right)$ velocity dispersions, compact tracers (such as $\mathrm{CO}$ ) result in Gaussian-like (non-doublehorned) profiles, as is indeed frequently seen in high-redshift observations. This leads to significantly different determinations of $M_{\mathrm{dyn}}$ if different distributions of the tracer material ("flat" versus "exponential") are considered. We determine at which radii the rotation curve reaches the rotation velocity corresponding to the velocity width, and find that for each tracer this happens at a well-defined radius: H I velocity widths typically originate at $\sim 5$ optical scale lengths, while $\mathrm{CO}$ velocity widths trace the rotation velocity at $\sim 2$ scale lengths. We additionally explore other distributions to take into account that $\mathrm{CO}$ distributions at high redshift likely differ from those at low redshift. Our models, while not trying to reproduce individual galaxies, define characteristic radii that can be used in conjunction with the measured velocity widths in order to define dynamical masses consistent with the assumed gas distribution.
\end{abstract}

Key words: galaxies: fundamental parameters - galaxies: ISM - galaxies: kinematics and dynamics radio lines: galaxies - submillimeter: galaxies

Online-only material: color figures

\section{INTRODUCTION}

Dynamical mass $\left(M_{\mathrm{dyn}}\right)$ is a fundamental property of galaxies at low and high redshift. In combination with measurements of the baryonic mass, it provides information about the amount of dark matter in galaxies. In the nearby universe, galaxies can, in principle, be well resolved and a rotation curve can be derived to accurately constrain $M_{\text {dyn }}$ as well as derive information about the distribution and importance of dark matter. This is frequently done by using interferometric observations to measure the Doppler-shift of the $21 \mathrm{~cm}$ emission line of neutral hydrogen (H I).

If a galaxy is not sufficiently resolved, then the derived rotation curve is no longer a true reflection of the mass distribution. However, even with spectroscopic data that are spatially unresolved or have a low spatial resolution, the amplitude of the rotational motions can still be used to derive an approximate value of the dynamical mass. Usually this is done by deriving the "global profile" of a galaxy: this shows the emission or flux of a galaxy as a function of apparent radial (Doppler) velocity. For local spiral galaxies with flat rotation curves and extended gas disks, the global profile takes the shape of the wellknown "double-horned profile," caused by the accumulation of Doppler-shifted emission at the projected velocities corresponding to the amplitude of the flat part of the rotation curve. In the absence of spatially resolved data, the global profile is usually the only option for estimating the dynamical mass. This situation occurs for single-dish H I surveys of the local universe (such as HIPASS or ALFALFA), but also for interferometric observations of galaxies at high redshifts.

The velocity width $W$ of the global profile gives an indication of (twice) the amplitude of the inclination-projected rotation of a galaxy. Widths are usually determined at $20 \%$ or $50 \%$ of the maximum value of the profile to avoid being affected by noisy regions in the spectrum, and are denoted as $W_{20}$ or $W_{50}$, respectively. An indicative dynamical mass can be defined as $M_{\text {dyn }}=\left[(W /(2 \sin i))^{2} R\right] / G$, where $W$ is the velocity width, ${ }^{5}$ $i$ is the inclination of the galaxy, $G$ the gravitational constant, and $R$ a representative radius. For a good estimate of the total dynamical mass, this radius should ideally be the maximum radius at which the tracer can still be observed. Some of the most common choices for $R$ are the radius of the H I disk or the optical disk. In the rest of this paper we use $W \equiv W_{50}$.

One important aspect that is often under-appreciated is that global profiles are not only determined by the rotation curve of a galaxy but also by the spatial distribution of the tracer gas within that galaxy. To date, most measurements of global profiles of low-redshift galaxies are based on $\mathrm{H}$ I measurements.

\footnotetext{
5 In practice, $W$ also needs to be corrected for instrumental effects such as the finite width of velocity channels. We will, however, ignore this in this paper.
} 
Neutral hydrogen has the advantage that it usually extends much farther out than the optical disk, thus tracing the rotation at large radii, and in many cases it comfortably reaches the flat part of the rotation curve. It also has a high area-covering factor and, to first order, constant surface density. These three properties together ensure that global profiles based on $\mathrm{H}$ I observations usually yield a good representation of the dynamics of galaxies. This is also the underlying reason why measurements of global H I profiles have been successfully used in evaluating dynamical masses and relations such as the Tully-Fisher (TF) relation (Tully \& Fisher 1977).

While Hi-based global profiles are therefore an important and convenient tool for low-redshift studies, this is not the case for higher redshifts. With current instrumentation, $\mathrm{H} \mathrm{I}$ emission is difficult to observe beyond the local universe. Only a relatively small number of galaxies have been observed directly in $\mathrm{H}$ I at larger redshifts out to $z \sim 0.2$ with significant investments of observing time (e.g., Verheijen et al. 2007). Even with large future telescopes such as the Square Kilometre Array, it is unlikely that large numbers of galaxies will be routinely observed in emission in $\mathrm{H}_{\mathrm{I}}$ beyond $z \sim 1$ without major investments in observing time (see, e.g., Abdalla et al. 2010).

Recent studies of the gas in galaxies at these higher redshifts have mainly used the CO line, which is the brightest tracer of the cold molecular gas component (see Carilli \& Walter 2013 for an overview). The $\mathrm{CO}$ line is intrinsically much brighter than the $\mathrm{HI}_{\mathrm{I}}$ line. However, an important difference with $\mathrm{H}_{\mathrm{I}}$ is that the $\mathrm{CO}$ distribution is known to be more compact, at least in detailed studies of nearby galaxies. Here the $\mathrm{CO}$ distribution rarely extends beyond the optical radius $R_{25}$. Also, in contrast with the almost constant Hi surface density, the CO surface density decreases exponentially with radius (e.g., Schruba et al. 2011; Leroy et al. 2009).

This immediately implies that $\mathrm{CO}$ can trace the rotation curve of a galaxy only out to a fraction of the radius that would, in principle, be traceable by $\mathrm{H}$. That is, for a given rotation curve, the global profile as measured in $\mathrm{H}$ I will look different from the one measured in $\mathrm{CO}$.

Finally, global profiles are also affected by the velocity dispersion of the gas. Low-redshift disk galaxies are very much dominated by rotation; the typical $\mathrm{H}_{\mathrm{I}}$ velocity dispersion of $\sim 11 \mathrm{~km} \mathrm{~s}^{-1}$ (Tamburro et al. 2009; Ianjamasimanana et al. 2012) is many times smaller than the rotation velocity, and the same is true of the low-redshift CO velocity dispersion (M. Mogotsi et al. 2014, in preparation; Caldú-Primo et al. 2013).

However, at higher redshifts, where there are more actively star-forming environments, the gas velocity dispersion is expected (and observed) to be much higher. Measurements of the ionized gas phase of main-sequence star-forming galaxies at $z \sim 1-3$ indicate dispersions of $30-100 \mathrm{~km} \mathrm{~s}^{-1}$ (e.g., Tacconi et al. 2013, and references therein). Direct measurements of the molecular gas dispersion at these redshifts are rare, but indicate dispersion values of $\sim 50 \mathrm{~km} \mathrm{~s}^{-1}$ (Swinbank et al. 2011) to $\sim 100 \mathrm{~km} \mathrm{~s}^{-1}$ (Hodge et al. 2012).

A fundamental assumption in the evaluation of a global profile is that the galaxy under consideration is rotation-dominated, i.e., the system is not undergoing a merger, is not accreting massive gas reservoirs that increase the dispersion, and is sufficiently massive that its kinematics are not entirely dominated by dispersion. At low redshift, most galaxies are rotation-dominated, and interacting systems are easily identified by their morphology. At high redshift, the situation is more difficult to assess from an ob- servational perspective. However, there is growing evidence that many of the $L^{\star}$ galaxies observed at higher redshifts are in fact rotation-dominated, even if based on their infrared luminosities one would classify them as ULIRGs (i.e., $L_{\mathrm{FIR}}>10^{13} L_{\odot}$; at low redshift ULIRGS are known to be interacting systems, but they constitute "main sequence" galaxies at high redshift).

There have been numerous attempts to quantify the kinematics of high-redshift galaxies, mostly using optical emission lines and integral field units on $10 \mathrm{~m}$ class telescopes. Early studies showed that $\sim 1 / 3$ of spatially resolved galaxies were dispersion-dominated (e.g., Weiner et al. 2006; Förster Schreiber et al. 2009; Law et al. 2009; Epinat et al. 2012). New observations of a sample of $z \sim 2.2$ galaxies with improved spatial resolution (using adaptive-optics-assisted imaging; Newman et al. 2013) have now shown that some of the galaxies thought to be dispersion-dominated in earlier studies are in fact rotating. These authors argue that the larger the size of the galaxy, the more rotation-dominated it is, as is expected (and found in the local universe) if galaxies have maximum rotation velocities that scale with size and have velocity dispersions that are independent of size. Newman et al. (2013) also highlight the issue of "beam smearing" which artificially increases the observed dispersion in semi-resolved measurements.

In summary, even though it is expected that a fraction of high-redshift galaxies is not rotation-dominated (due to mergers, etc.), recent observational evidence clearly demonstrates that a significant fraction of this galaxy population is rotating. In this paper we therefore assume galaxies that are rotation-dominated, down to masses where the dispersion becomes a significant contributor. We quantify how the choice of gas tracer and its intrinsic distribution (from constant surface density to ultracompact distributions) changes the estimated dynamical mass of a given galaxy using a number of galaxy rotation curve templates.

In Section 2 we describe the method we use to derive the profiles and the assumptions we make in that process. In Section 3, we discuss the resulting velocity widths and their impact on galaxy scaling relations. Section 4 deals with the problem of finding a length scale over which dynamical masses are computed. We summarize our conclusions in Section 5.

\section{GENERATING GLOBAL PROFILES}

Here we briefly describe the method used to generate the global profiles. In Section 2.1, we first summarize the procedure used to calculate global profiles given an input rotation curve and a radial gas surface density distribution. Section 2.2 describes the properties of the sample of rotation curves we use in this analysis. Section 2.3 addresses our choices for the radial surface density profiles.

\subsection{Profiles}

We follow the method described in Obreschkow et al. (2009) and briefly summarize the main features here. The line profile of an edge-on, optically thin, filled, flat ring with a constant circular velocity $V_{c}$ and a luminosity of unity can be derived as follows. The apparent observed (radial) velocity $V_{\text {obs }}$ of a point on the ring is given by $V_{\mathrm{obs}}=V_{c} \sin \gamma$, where $\gamma$ is the angle with the line of sight. Evaluating the change in $V_{\text {obs }}$ as a function of $\gamma$, the normalized edge-on line profile of the ring can be written 
(following the notation from Obreschkow et al. 2009) as

$$
\tilde{\psi}\left(V_{\mathrm{obs}}, V_{c}\right)= \begin{cases}\frac{1}{\pi \sqrt{V_{c}^{2}-V_{\mathrm{obs}}^{2}}} & \text { if }\left|V_{\mathrm{obs}}\right|<V_{c} \\ 0 & \text { otherwise. }\end{cases}
$$

This equation does not take into account the velocity dispersion of the gas. A velocity dispersion $\sigma$ can be incorporated in the above description as a convolution of $\tilde{\psi}$ with a Gaussian with dispersion $\sigma$, yielding a line profile $\psi$. This procedure also softens the discontinuities $\tilde{\psi}$ has for $\left|V_{\text {obs }}\right| \rightarrow V_{c}$. We assume the velocity dispersion to be isotropic.

Obreschkow et al. (2009) take into account flux normalizations of the profiles. As we are only concerned with the relative changes of the line profiles, and line widths are not affected by normalizations, we ignore these normalization factors here.

So far, we have assumed rings with a luminosity of unity. The distribution of the gas in a galaxy can be taken into account by multiplying $\psi$ of each ring with the luminosity (or gas mass) in that ring. The total global profile $\psi\left(V_{\mathrm{obs}}\right)$ can be calculated as

$$
\psi\left(V_{\mathrm{obs}}\right)=2 \pi \sum_{r=0}^{R} r \Delta r \Sigma(r) \psi\left(V_{\mathrm{obs}}, V_{c}(r)\right),
$$

where $\Delta r$ is the width of a ring (with $\Delta r$ chosen to be small compared to the extent of the galaxy), $\Sigma(r)$ is the azimuthally averaged radial profile of the gas distribution, $V_{c}(r)$ the circular velocity curve, which is usually taken to be equal to the rotation curve, and $R$ is the maximum measured radius of the gas distribution. Note that we do not take into account optical depth effects.

The line profile of a non-edge-on galaxy can be calculated by multiplying $\tilde{\psi}$ in Equation (1) with $\sin i$ before convolving with the velocity dispersion Gaussian. The procedure described here thus allows calculation of global profiles for any given combination of surface density profile and rotation curve and arbitrary values of inclination and velocity dispersion.

\subsection{Rotation Curves}

To calculate global profiles comparable to those typically observed (at least at low redshift), we need representative rotation curves as input, preferably over a comprehensive range of galaxy masses and luminosities. We here use a set of template rotation curves from Catinella et al. (2006). They used long-slit optical rotation curves and $I$-band photometry of $\sim 2200$ low-redshift disk galaxies to construct average template rotation curves for 10 separate luminosity classes, spanning six magnitudes in $I$-band luminosity, ranging from $M_{I}=-23.8$ to $M_{I}=-19.0$ (approximately equal to a range from $M_{B} \sim$ -22.0 to $M_{B} \sim-17.2$, assuming $B-I \sim 1.8$; cf. de Jong 1996 b, their Figure 10 and Table 2). For convenience we denote these luminosity classes with a numerical code $t$, with $t=0$ corresponding to the brightest luminosity class and $t=9$ to the faintest luminosity class (see also Table 1).

The template curves as presented in Catinella et al. (2006) are expressed in terms of the exponential disk scale length $h$ and in all cases extend out to $\sim 5 h$. Catinella et al. (2006) parameterize these curves using the so-called polyex model (Giovanelli \& Haynes 2002),

$$
V_{\mathrm{PE}}(r)=V_{0}\left(1-e^{-r / r_{\mathrm{PE}}}\right)\left(1+\frac{\alpha r}{r_{\mathrm{PE}}}\right) .
$$

\begin{tabular}{|c|c|c|c|c|c|c|c|}
\hline (1) & $\begin{array}{c}M_{I} \\
(\mathrm{mag}) \\
(2)\end{array}$ & $\begin{array}{c}\Delta M_{I} \\
(\mathrm{mag}) \\
(3)\end{array}$ & $\begin{array}{c}V_{0} \\
\left(\mathrm{~km} \mathrm{~s}^{-1}\right) \\
(4)\end{array}$ & $\begin{array}{c}r_{\mathrm{PE}} / h \\
(5)\end{array}$ & $\begin{array}{l}\alpha \\
(6)\end{array}$ & $\begin{array}{c}h \\
(\mathrm{kpc}) \\
(7)\end{array}$ & $\begin{array}{c}V_{\max } \\
\left(\mathrm{km} \mathrm{s}^{-1}\right) \\
(8)\end{array}$ \\
\hline 0 & -23.8 & 0.4 & 270 & 0.37 & 0.007 & 10.4 & 294 \\
\hline 1 & -23.4 & 0.4 & 248 & 0.40 & 0.006 & 8.6 & 267 \\
\hline 2 & -23.0 & 0.4 & 221 & 0.48 & 0.005 & 7.2 & 235 \\
\hline 3 & -22.6 & 0.4 & 188 & 0.48 & 0.012 & 6.0 & 217 \\
\hline 4 & -22.2 & 0.4 & 161 & 0.52 & 0.021 & 5.0 & 201 \\
\hline 5 & -21.8 & 0.4 & 143 & 0.64 & 0.028 & 4.1 & 184 \\
\hline 6 & -21.4 & 0.4 & 131 & 0.73 & 0.028 & 3.4 & 163 \\
\hline 7 & -21.0 & 0.4 & 116 & 0.81 & 0.033 & 2.9 & 144 \\
\hline 8 & -20.4 & 0.8 & 97 & 0.80 & 0.042 & 2.1 & 128 \\
\hline 9 & -19.0 & 2.0 & 64 & 0.72 & 0.087 & 1.1 & 102 \\
\hline
\end{tabular}

Table 1

Properties of Template Rotation Curves

Notes. (1) Luminosity $t$-type. (2) Average luminosity of galaxies in this bin. (3) Spread in luminosity of galaxies in this bin. (4) Velocity amplitude of polyex fit (Equation (3)). (5) Steepness of inner polyex fit rotation curve, expressed in scale lengths. (6) Slope of outer polyex fit rotation curve. (7) Exponential disk scale length in kpc. (8) Maximum velocity of polyex fit rotation curve. Columns 2-6 are from Catinella et al. (2006).

Here $V_{0}, r_{\mathrm{PE}}$, and $\alpha$ describe the amplitude of the rotation curve, the steepness of the inner rise of the curve, and the slope of the outer part, respectively. The average luminosities of each luminosity class and the parameters of the corresponding polyex fits, taken from Catinella et al. (2006), are listed in Table 1. For a full discussion of curves and fits we refer to Catinella et al. (2006). In the following we will be adopting the polyex fits as our fiducial template curves. This has the advantage that smallscale variations in the rotation curve do not affect the results. The curves and polyex fits with radii expressed in scale lengths are shown in the left panel in Figure 1.

We will be varying the distribution of the gas tracer in our models and therefore need to define a representative reference length scale for each template rotation curve. We here choose the optical exponential disk scale length $h$, which, as noted, is also the unit of radius of the Catinella et al. (2006) template curves.

It is useful to also express the rotation curve radii in kiloparsecs, and we calculate values for the scale lengths $h$ as follows. First, we assume that the template galaxies are Freemandisks (i.e., that they have a central disk surface brightness of $\mu_{0}^{B}=21.65 \mathrm{mag} \operatorname{arcsec}^{-2}$ ), which is a reasonable assumption for this range in luminosity (see, e.g., de Jong 1996a). Note that as we are ignoring normalization factors in this analysis, the exact choice of central surface brightness value is not critical. Using an average color for these galaxies of $B-I \sim 1.8$ (as also used above), we calculate the Freeman-value in the $I$-band. By assuming exponential disks, we can use $L_{I}=2 \pi \Sigma_{0}^{I} h^{2}$ to calculate the exponential scale length $h$. Here $L_{I}$ and $\Sigma_{0}^{I}$ are the $I$-band luminosity and Freeman central surface brightness value, expressed in $L_{\odot}$ and $L_{\odot} \mathrm{pc}^{-2}$, respectively. The scale lengths $h$ that are derived for each template rotation curve are listed in Table 1. The right panel in Figure 1 shows the resulting template curves and polyex fits with the radii in kpc scaled using the appropriate scale lengths. The left panel in Figure 1 shows that all curves extend to $\sim 5 h$; the right panel shows that the decrease in scale length from high to low luminosity (or, equivalently, galaxy mass) results in the curves extending less far (in terms of kiloparsecs) as luminosity decreases.

A maximum radius of $5 h$ agrees well with the typical sizes of $\mathrm{H}$ I disks in nearby disk galaxies. For a sample of 108 nearby 



Figure 1. Template rotation curves from Catinella et al. (2006; gray symbols). The polyex fits are overplotted as the black curves. The average luminosity corresponding to each template curve is given to the right of the terminal velocity of each curve. The left-hand panel shows the curves plotted with the radius expressed in scale lengths $h$. The right-hand panel shows the same curves with the radius in kpc. These are derived by multiplying the radial scale in units of scale length by the value of $h$ as derived in the text and given in Table 1.

gas-rich disk galaxies, Broeils \& Rhee (1997) find a relation between the $\mathrm{H}_{\mathrm{I}}$ radius $R_{\mathrm{H}_{\mathrm{I}}}$ (defined at the $1 M_{\odot} \mathrm{pc}^{-2}$ column density level) and the optical radius $R_{25}$ (defined at the $25 \mathrm{~B}$ mag $\operatorname{arcsec}^{-2}$ level) of $R_{\mathrm{HI}} \sim(1.70 \pm 0.16) R_{25}$. For nearby disk galaxies $R_{25} \simeq 3.2 h$, yielding an $\mathrm{H}$ I radius $R_{\mathrm{HI}} \sim(5.4 \pm 0.5) h$. The $5 h$ maximum radius used here is therefore a reasonable approximation of that found in real disk galaxies.

The rotation curves described here are derived from local galaxies. Are they also applicable to high-redshift galaxies? As described in Section 1, there is good evidence that a substantial fraction of galaxies at higher redshifts are dominated by rotation. In addition, the $\mathrm{TF}$ relation, one of the most well-defined relations involving rotating disk galaxies, seems to be in place already by $z \sim 1.7$ (e.g., Miller et al. 2013), and there are indications it may already exist by $z \sim 3$ (e.g., Gnerucci et al. 2011), though with an offset in stellar mass. Galaxies with well-defined rotation curves with a range in maximum rotation velocities overlapping with that observed locally can thus be presumed to exist at higher redshifts as well. We will later show that the global profiles are only moderately sensitive to the exact shape of the rotation curve.

Whether the gas distributions at higher redshifts are similar to those found locally is an open question. However, as described in the following subsection, we circumvent this by calculating global profiles for a large range of surface density profiles.

\subsection{Radial Gas Distributions}

We adopt the scale lengths $h$ derived above as the "standard" values appropriate for each luminosity class $t$. That is, if in a template galaxy the gas tracer is distributed like the stars, it also has a scale length $h$. In evaluating global profiles, we vary the scale length of the gas distribution with respect to this reference value $h$. For each template rotation curve (or luminosity bin $t$ ) we assume gas distributions with scale lengths $\ell=h / 4, h / 2, h$, $2 h$ and $4 h$, as well as a constant ("flat") radial surface density, the latter corresponding to the situation typically found in $\mathrm{HI}$ disks in galaxies.

In addition, we evaluate the distribution given in Schruba et al. (2011, hereafter S11) who found that the CO radial profiles in a number of nearby disk galaxies could be described as an exponential with $\ell \simeq 0.2 R_{25}$. Again using that $R_{25} \simeq 3.2 h$, we can describe the empirical S11 distribution using a scale length $\ell_{\mathrm{S} 11}=0.64 h$.

In constructing the global profiles, we take into account that the template rotation curves themselves terminate at $\sim 5 h$. Models which, due to a large value of $\ell$, give rise to very extended gas distributions are truncated at $R=5 h$, thus taking into account the typical "largest radius" accessible in observations of (nearby) galaxies.

In Appendix A we compare a number of our analytically derived global profiles with those derived from more computationally intensive numerical, three-dimensional models. We find very good agreement and proceed to use the analytical profiles in the rest of this paper.

The large range in radial density profiles presented here enables evaluation of profiles based on a situation that deviates from what is found locally. If, for example, the observed $\mathrm{CO}$ distribution at high redshift is more compact than what is observed at low redshift (because high-J CO transitions have been observed, or because of a radially changing CO-to- $\mathrm{H}_{2}$ conversion factor, e.g., due to steep metallicity gradients), then it is easy to calculate the profile for a smaller $\ell$-value. We emphasize here that we are not attempting to provide a definitive description of a high-redshift galaxy. Rather, we evaluate profiles covering a range in maximum rotation velocities and gas densities that overlaps with what is found observationally. A comparison with these observations should therefore be made under the following assumptions: (1) galaxies are virialized and dominated by rotation, (2) galaxies do not suffer from major interactions and/or asymmetries (though the latter will have only a minor impact on $W_{50}$ ), (3) rotation curves are to first 

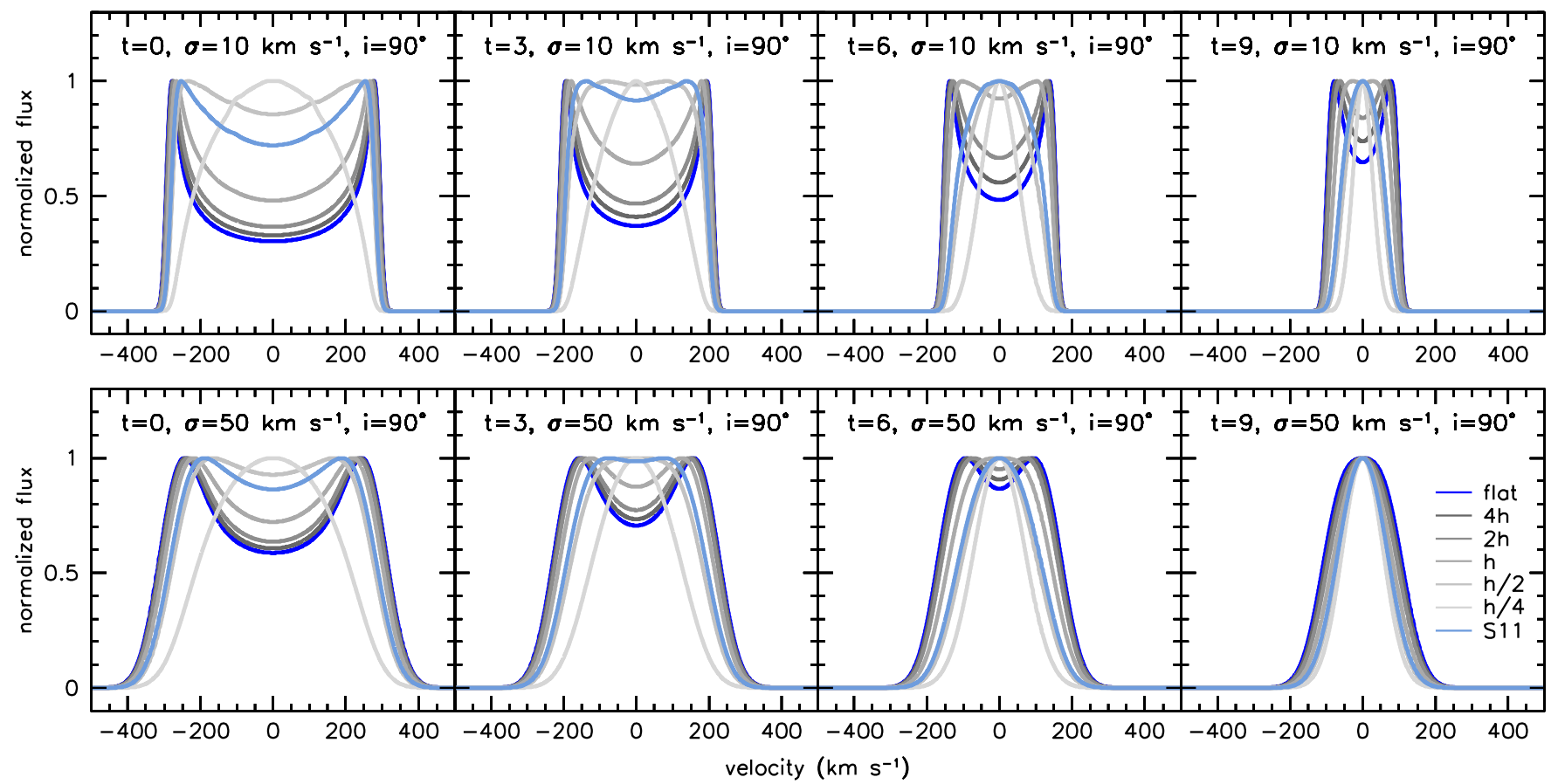

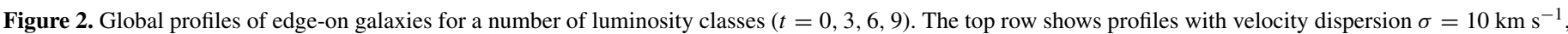

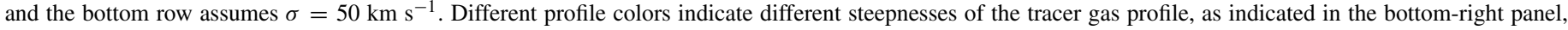

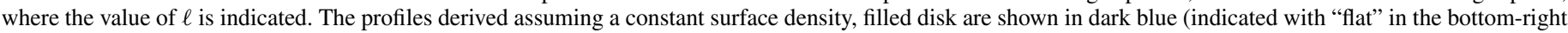

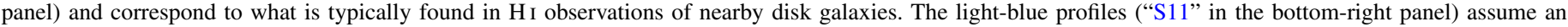
exponential disk with scale length $\ell=0.64 h$, as found by Schruba et al. (2011) for nearby galaxies.

(A color version of this figure is available in the online journal.)

order independent of redshift (as hinted at by the existence of a high-redshift TF relation), and (4) the range in gas density profiles presented here covers that found in real galaxies.

Also note that the surface density of the tracer component is not relevant for our models, as we only evaluate normalized global profiles. In order to model more compact or more extended gas distributions only the scale length $\ell$ needs to be adjusted.

\section{RESULTS}

\subsection{Comparing Velocity Widths}

For each of the template rotation curves ( $t$-types), we calculate global profiles, assuming exponential radial gas density profiles with scale lengths $\ell=(h / 4, h / 2, h, 2 h, 4 h)$, where $h$ is the "standard" scale length for each template galaxy class as listed in Table 1. We also calculate global profiles for a constant surface density filled disk and an exponential disk with an S11 scale length $\ell_{\mathrm{S} 11}=0.64 h$. Gas distributions are in all cases truncated at a radius of $5 h$.

We calculated many sets of global profiles assuming different combinations of inclination and velocity dispersion. Here we concentrate on the profiles of edge-on galaxies with $\sigma=10$ and $50 \mathrm{~km} \mathrm{~s}^{-1}$, representative of velocity dispersions typically found in low- and high-redshift galaxies, respectively. Appendix B presents profiles at inclinations of $60^{\circ}$ and $30^{\circ}$.

Figure 2 shows the profiles for $t=(0,3,6,9)$ edge-on galaxies, assuming $\sigma=10 \mathrm{~km} \mathrm{~s}^{-1}$ (top row) and $50 \mathrm{~km} \mathrm{~s}^{-1}$ (bottom row) velocity dispersion and using the range of $\ell$ definitions given above. The steepness of the gas profiles is indicated by the color of the profile (as indicated in the bottom-right panel).
In Figure 2, we see that for the low-t, low-dispersion, constant-surface-density models the double-horned signature of the profiles is very pronounced. In contrast, for very steep gas profiles, where the density has already become negligible at radii where the rotation velocity has not reached the flat part yet, the horns disappear, resulting in a more Gaussian profile. This is an effect that we see for all parameter combinations. It is most pronounced at higher $t$-values, where due to the slower rising rotation curves, the horns already disappear for moderately steep gas profiles.

As the value of the velocity dispersion increases (bottom row of Figure 2), we see that the horns are less pronounced, with the profiles becoming Gaussian or flat-topped over a large range of parameter combinations. This could be an explanation for why many $\mathrm{CO}$ spectra of galaxies at high redshift do not exhibit the double-horn shape. For example, the majority of the quasar host galaxies show profiles that are well fit by just a simple Gaussian (e.g., Riechers et al. 2011). This is also the case for most of the submillimeter galaxies (e.g., Bothwell et al. 2013). Recent large samples of main sequence galaxies have also revealed predominantly Gaussian CO profile shapes (e.g., Tacconi et al. 2013). The double-horned profiles that are observed at high redshifts are most often associated with massive galaxies, which must have steeply rising rotation curves or very extended molecular gas disks to ensure that the double-horned signature remains visible. For example, the double-horned $\mathrm{CO}$ profiles shown in Daddi et al. (2010) belong to high-redshift galaxies with velocity widths $400-600 \mathrm{~km} \mathrm{~s}^{-1}$, which, in terms of velocity width, are comparable to the $t=0$ or $t=1$ galaxies as discussed here (cf. Figure 2).

In the majority of cases, the apparent velocity width of the profile becomes discernibly smaller as the steepness of the gas surface density profile increases. The change can be a significant 


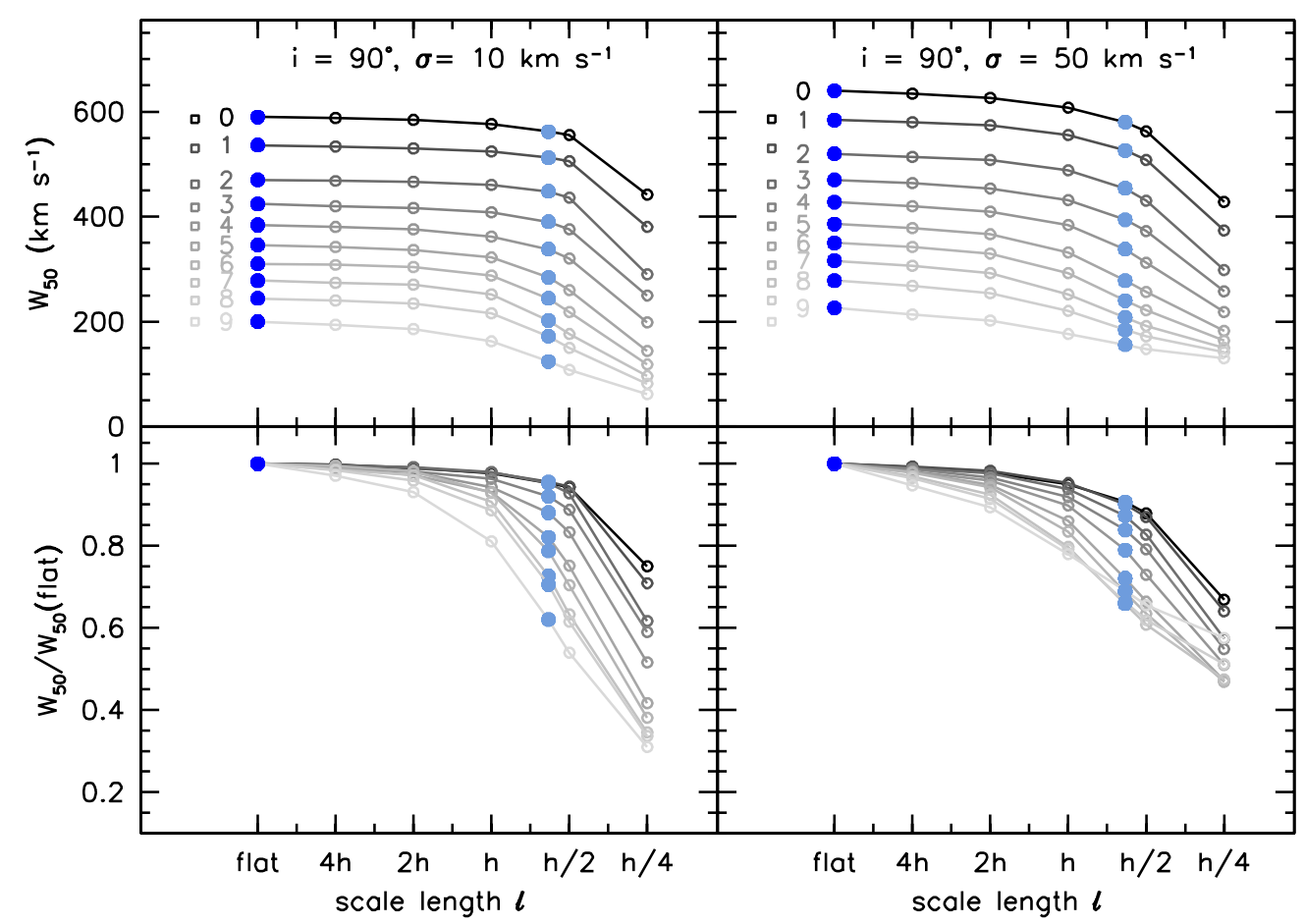

Figure 3. $W_{50}$ values for velocity dispersions of $10 \mathrm{~km} \mathrm{~s}^{-1}$ (left) and $50 \mathrm{~km} \mathrm{~s}^{-1}$ (right) as a function of profile steepness (scale length $\ell$ ) for edge-on galaxies. Gray scales represent the $t$ type (also indicated in the top panels). Dark-blue indicates flat density profiles ("H I"), and light-blue indicates S11 profiles ("CO"). For comparison, the small squares in the top panels indicate the values for the flat density profiles, assuming a zero velocity dispersion.

(A color version of this figure is available in the online journal.)

fraction of the velocity width, especially for the lower luminosity galaxies. This is quantified in Figure 3, where we show how $W_{50}$ changes as a function of the gas distribution ${ }^{6}$ (shown here for only for edge-on galaxies; other inclinations are shown in Appendix B).

The values of $W_{50}$ are largely unaffected for gas distributions that have scale lengths $\ell>h$ (i.e., extended distributions). For smaller values of $\ell$ (compact distributions), the measured values decrease rapidly, especially for the less luminous galaxies (high $t$-values). For the $\mathrm{S} 11$ profile, the ratio of the measured $W_{50}$ value and the value for a constant surface density disk decreases from slightly less than unity for $t=0$ to $\sim 0.6$ for $t=9$. These trends are visible for both values of the velocity dispersion. Note that for $\sigma=50 \mathrm{~km} \mathrm{~s}^{-1}$, we see a different behavior for the smallest $\ell$ values. In this case the velocity dispersion dominates the measured $W_{50}$ values, and we see a flattening of the decrease in $W_{50}$ as a function of decreasing $\ell$.

\subsection{The Tully-Fisher Relation}

These findings have direct consequences for the interpretation of scaling relations involving a velocity width. Perhaps the most famous of these is the TF relation (Tully \& Fisher 1977). This relation between the luminosity and velocity line width of galaxies has been used as a distance indicator, but the apparent universality of the TF relation has meant that it has also become an important part of studies of the dark and baryonic matter content of galaxies (e.g., McGaugh et al. 2000; McGaugh 2012).

The TF relation is most often studied using $\mathrm{H} \mathrm{I}$ as a tracer for the dynamics. Here we investigate whether the parameters of

\footnotetext{
6 For most profiles this width is unambiguously defined. In cases where the $50 \%$ level crosses the horns, we define $W_{50}$ to be the difference between the two outermost crossings (i.e., the ones with the highest and lowest velocities).
}

the TF relation, in particular the slope, are sensitive to changes in the radial profile of the tracer gas. Aspects of this question were already investigated in papers by Tutui \& Sofue (1999) and Lavezzi \& Dickey (1998). Tutui \& Sofue (1999) already note the effect that the different $\mathrm{CO}$ and $\mathrm{H}$ I distributions will have.

In Figure 4 (top row), we show the TF relations derived using our measured velocity widths for the various assumptions on the steepness of the tracer gas profile and a velocity dispersion $\sigma=10 \mathrm{~km} \mathrm{~s}^{-1}$. We use the absolute $I$-band magnitudes corresponding to each $t$-type, as given in Table 1. For comparison, the TF relation as defined by the rotation velocities at the outermost point of the template rotation curves is also shown. Note that Verheijen (2001) finds an I-band TF slope of $3.8 \pm 0.1$, based on global profile velocity widths in an $\mathrm{H}$ i study of the TF relation of regular disk galaxies in the Ursa Major cluster (the value quoted here applies to their "DE sample"). In general, $\mathrm{H}$ I-based studies of the TF relation tend to find slopes $\approx 4$ (e.g., McGaugh et al. 2000; Verheijen 2001).

From Figure 4 it is clear that the slope of the TF relation decreases as the steepness of the tracer gas profile increases. Moderately steep density profiles already result in a significant change in the slope.

Note that the TF relation based on the flat density profile shows a change in the slope around $M_{I} \sim-21$. We therefore determine two values for the slope of each $\mathrm{TF}$ relation. The first one is determined using the full luminosity range, i.e., all $t$-types. The second one only uses the galaxies with $M_{I}<$ -20.5 , i.e., only $t=0-7$ (the galaxies above the break). Figure 4 (right) shows the resulting TF-slopes as a function of the steepness of the gas profile. For the flat and extended density profiles we find TF-slopes that are in reasonable agreement with those found in the detailed TF study by Verheijen (2001) 

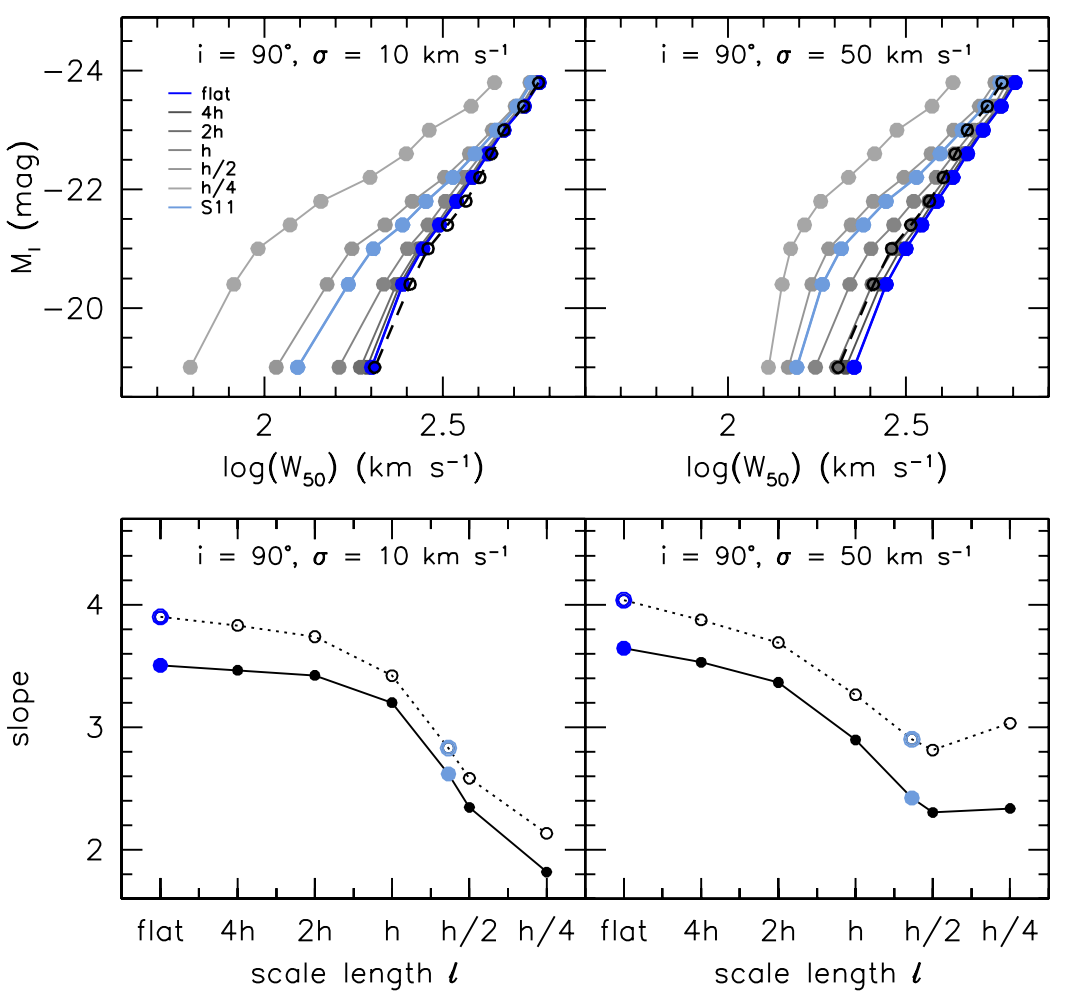

Figure 4. Top-left: TF relations for various gas tracer profile steepnesses assuming $\sigma=10 \mathrm{~km} \mathrm{~s}^{-1}$. Steepnesses are indicated in the panel. The black dashed line with open circles represents the TF relation derived using the outer velocity in the rotation curves. Bottom-left: fitted slopes of the TF relations. Dotted lines and open symbols show slopes of the full TF relations $(t=0-9)$, and full lines and filled symbols show slopes derived using only points with $M_{I}<-20.5(t=0-7)$. Two measures of the slope are used due to the break in the TF around $M_{I} \sim-21$. Top-right: same as top-left, but for $\sigma=50 \mathrm{~km} \mathrm{~s}{ }^{-1}$. Bottom-right: same as bottom-left, but for $\sigma=50 \mathrm{~km} \mathrm{~s}^{-1}$.

(A color version of this figure is available in the online journal.)

mentioned earlier, but note that the TF-slope starts to decrease around $\ell=h$. The slope of the TF relation derived assuming S11 density profiles has decreased by a full unit compared to the value found for flat density profiles. The slope thus depends on the steepness of the density profile, and for the models considered here a "CO"-TF would be less steep than an "H I"TF. This conclusion applies to any density profile steeper than $\ell \sim h$.

Results for a velocity dispersion of $50 \mathrm{~km} \mathrm{~s}^{-1}$ are similar (Figure 4, bottom-row), with the one difference that at low line widths the dynamics are clearly dominated by the dispersion. Nevertheless, also here, a TF measured in $\mathrm{CO}$ would have a more shallow slope than an H I-based TF.

\section{COMPARING DYNAMICAL MASSES}

When velocity widths are used to estimate dynamical masses, the differences due to the different tracer gas profiles directly propagate into the mass estimates through the square of the difference via $M_{\text {dyn }} \sim W_{50}^{2} \times R$. A second complication is that a radius $R$ is needed for the calculation of the dynamical mass. In practice, when one has to resort to using $W_{50}$ for dynamical mass estimates, this usually means that the observations of the gas component are unresolved or, at best, semi-resolved, and $R$ cannot be measured directly. It is therefore customary to assume a radius that is deemed appropriate for a given tracer. In the case of high-redshift $\mathrm{CO}$ observations of main sequence galaxies, this is typically chosen to be the optical radius, based on the results found for nearby galaxies (e.g., Leroy et al. 2008, 2013) and the few marginally resolved $\mathrm{CO}$ measurements at high redshift (e.g., Daddi et al. 2010; Tacconi et al. 2013). In dealing with these observations, one has to be aware of possible mismatches between the distribution of the tracer and the assumed radius for the dynamical mass.

\subsection{Tests Using Rotation Curves}

To address the problem of choosing the appropriate radius, we will here use our models of the global profiles to identify radii that are intrinsic to the tracer distribution used. In combination with the global profile, this radius can then be used to give a consistent estimate of the dynamical mass assuming a radius appropriate for the chosen gas distribution.

In the following we will, for brevity, only discuss the cases for flat ("H I"), $\ell=h$ ("exponential"), and $\ell=\ell_{\mathrm{S} 11}$ ("CO") density profiles, but results for other density profiles can be derived in a similar manner. For each of the three surface density distributions, we identify in the template rotation curves those radii that show the same rotation velocity as the $W_{50} / 2$ values of the corresponding global profile. As the rotation curve velocities do not contain a dispersion component, we compare with $W_{50}$ values derived from global profiles we calculate assuming zero dispersion, hereafter denoted as $W_{50}^{0}$. We return to the impact of the velocity dispersion at the end of this subsection.

Figure 5 shows the template rotation curves again, with the radii given in units of $h$. In the figure, we also indicate with arrows the measured $W_{50}^{0} / 2$ values for each combination of $t$-type and the three density profiles (flat, exponential and S11). We also indicate the radii at which these velocities occur. It is immediately clear that each type of density profile is associated with its own distinct range in radii. For the flat density profiles, the $W_{50}^{0}$ value measured is associated with a radius of 




Figure 5. Template rotation curves with radii expressed in scale lengths. See Figure 1 for luminosities and $t$-type numbering. The arrows indicate the $W_{50}^{0} / 2$ values assuming zero dispersion for the flat (blue), exponential (gray), and S11 (light-blue) surface density profiles for each of the $t$-types as indicated to the right of the arrows. The filled colored circles overplotted on top of the curves indicate the radii where the respective $W_{50}^{0} / 2$ values occur, using the same colors as the corresponding arrows.

(A color version of this figure is available in the online journal.)

$(4.6 \pm 0.2) h$; the exponential profile with a radius of $(3.1 \pm 0.3) h$, and the S11 profile with $(1.9 \pm 0.4) h$.

A $W_{50}^{0}$ velocity width determined in $\mathrm{H}_{\mathrm{I}}$ (i.e., with a flat density profile) is thus measuring the rotation velocity that is typically found at $\sim 5 h$, and the latter is therefore the appropriate radius to use in calculating the dynamical mass. Assuming an S11 profile instead (corresponding to a low-redshift CO measurement), will yield a different velocity width due to the steeper density profile; here the appropriate radius is $\sim 2 h$. Under the assumptions made here, velocity widths measured in $\mathrm{H}$ I are thus representative of the dynamical mass enclosed within a $\sim 5 h$ radius, while $\mathrm{CO}$ measurements trace the dynamical mass enclosed within a $\sim 2 h$ radius. The $\mathrm{CO}$ distribution and radii discussed here apply, strictly speaking, only to local galaxies. Should the CO distribution in high-redshift galaxies be different, then an appropriate, different value of $\ell$ can be chosen and corresponding radii derived.

Dynamics of real galaxies contain a dispersion term: for the three density profiles we discuss here we have calculated the ratio of velocity widths that include a dispersion and the zerodispersion velocity widths. These ratios are plotted in Figure 6. For the flat density profile, corrections rise approximately linearly with dispersion, until they reach values of $\sim 20 \%$ at $\sigma=$ $100 \mathrm{~km} \mathrm{~s}^{-1}$ for all but the latest $t$-types where the corrections are much higher. The exponential density profiles shows somewhat smaller corrections than the flat density profile, peaking at $\sim 15 \%$, with again the late $t$-types showing larger corrections. The S11 profile yields larger corrections overall, with the latest types already diverging at intermediate dispersions. Note that for the S11 profiles, and to a lesser extent also the exponential profiles, we find some ratios that are slightly less than unity. This is a real effect, with the larger dispersions moving flux to

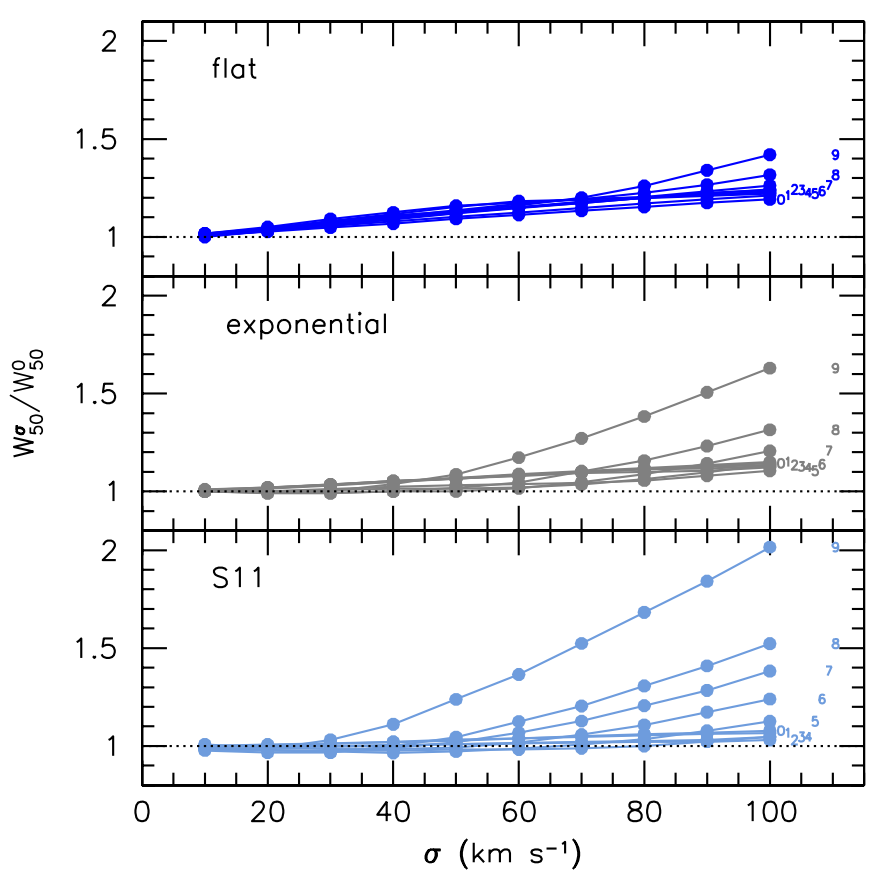

Figure 6. Ratio of the velocity widths that include velocity dispersion (as indicated on the horizontal axis) and the zero-dispersion velocity widths. From top to bottom are shown the ratios for the flat, exponential, and S11 density profiles. In each panel curves are shown for each $t$-type, as indicated by the numbers in the right-most part of the plot. Corrections more than $\sim 15 \%-20 \%$ should be treated with care, as they indicate the global profile is not dominated by rotation.

(A color version of this figure is available in the online journal.)

more extreme velocities, giving the central profiles a slightly more narrow core.

The larger corrections with increasing density profile steepness show how the steep density profiles ensure that a relatively small part of the inner rotation curve is sampled, causing the velocity dispersion to have a higher impact. Profiles with corrections that deviate strongly from the locus of curves shown in Figure 6 are thus unlikely to retain much information about the rotation of the galaxy, as can also be deduced from comparing profile shapes in Figure 2. Corrections higher than $~ 15 \%-20 \%$ should therefore be treated with caution as the profile retains little rotational information.

Given an observed global profile and assumed mass density profile, one can therefore estimate a representative dynamical mass using the velocity-dispersion-corrected velocity width and the corresponding characteristic radius.

\subsection{Tests Using Global Profiles}

In the previous subsection we derived the characteristic radii using the input rotation curves. This is an illustrative way of defining and locating the characteristic radii, but we can also derive these directly from the global profile. This has the advantage that the velocity dispersion can be taken into account directly. To derive characteristic radii from the profile, recall Equation (2). This describes the value of the global profile at velocity $V_{\text {obs }}$ as the weighted sum of the fluxes that the individual line profiles corresponding to each radius $r$ produce at $V_{\text {obs. }}$. The flux in each ring, $2 \pi r \Delta r \Sigma(r)$, thus serves as the weighting factor for $\psi$ of each ring. We can now calculate the weighted mean radius at each $V_{\mathrm{obs}}$ and identify for each value of $V_{\mathrm{obs}}$ the radius which, in terms of weighted mean average, is the dominant contributor to the total flux at that velocity. This then 


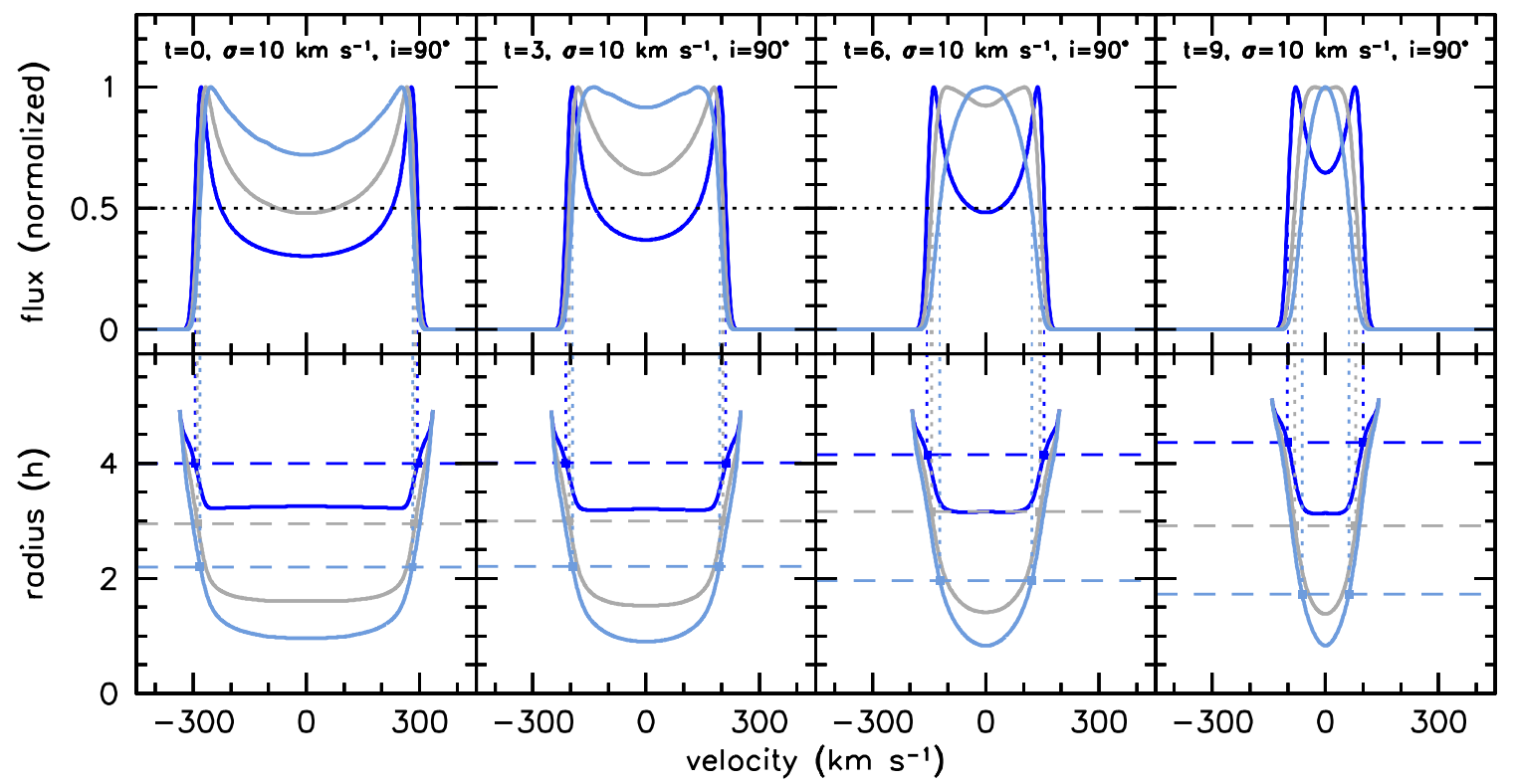

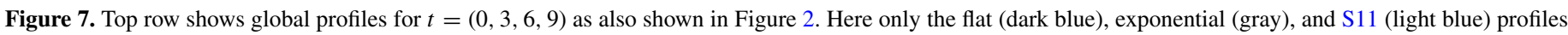

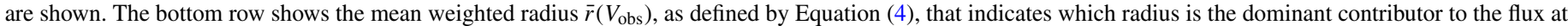

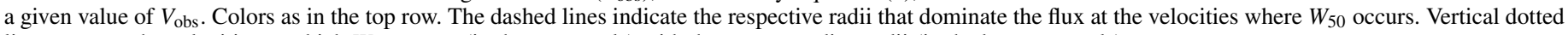
lines connect the velocities at which $W_{50}$ occurs (in the top panels) with the corresponding radii (in the bottom panels).

(A color version of this figure is available in the online journal.)

makes it possible to determine the characteristic radii that give rise to the flux at the velocities that define $W_{50}$.

Defining the weights $w_{r}=2 \pi r \Delta r \Sigma(r) \psi\left(V_{\mathrm{obs}}, V_{c}(r)\right)$, the weighted mean radius $\bar{r}$ for each value of $V_{\text {obs }}$ is given by

$$
\bar{r}\left(V_{\mathrm{obs}}\right)=\frac{\sum_{r=0}^{R} w_{r} r}{\sum_{r=0}^{R} w_{r}} .
$$

In Figure 7 we plot the radii $\bar{r}\left(V_{\mathrm{obs}}\right)$ for the $\sigma=10 \mathrm{~km} \mathrm{~s}^{-1}$ profiles that are also shown in Figure 2. We also indicate the radii at which $W_{50}$ occurs. We see a similar trend as the one in Figure 5, where the more compact density profiles have smaller characteristic radii. We can, analogous to Equation (4), derive the weighted standard deviation of the radii contributing to $V_{\mathrm{obs}}$ using

$$
s\left(V_{\mathrm{obs}}\right)=\sqrt{\frac{\sum_{r=0}^{R} w_{r}\left(r-\bar{r}\left(V_{\mathrm{obs}}\right)\right)^{2}}{\frac{N-1}{N} \sum_{r=0}^{R} w_{r}}},
$$

where $\bar{r}$ is the weighted mean from Equation (4), and $N$ is the number of profiles of individual rings (radii; as defined in Equation (1)) that have a non-zero contribution to the total global profile at $V_{\text {obs }}$ (as defined in Equation (2)). For our models, $N$ will be large and $(N-1) / N \approx 1$.

In Figure 8 (top-left panel) we plot the mean radii $\bar{r}$ where $W_{50}$ occurs (i.e., the equivalents of the radii indicated in Figure 5) as a function of $t$-type assuming $\sigma=10 \mathrm{~km} \mathrm{~s}^{-1}$. We also show the corresponding standard deviation $s$, but again only present results for the flat, exponential and S11 density profiles. The characteristic radii for each density profile are well separated for all $t$-values. The average values for the radii we find are $(4.1 \pm 0.2) h$ for the flat profile, $(3.0 \pm 0.1) h$ for the exponential profile, and $(2.0 \pm 0.2) h$ for the S11 profile (not taking into account the standard deviations; the quoted uncertainties are the uncertainties in the mean). These radii agree very well with those derived based on the rotation curves (see Section 4.1).
Results for $\sigma=50 \mathrm{~km} \mathrm{~s}^{-1}$ are similar (Figure 8, bottom-left panel).

\subsection{Dependence on Rotation Curve Shape}

We test how robust the characteristic radii are against changes in the shape of rotation curve. One would expect that, to first order, the velocity width derived from a global profile does not depend on the precise shape of the rotation curve, but is determined mainly by the amplitude of the rotation, the steepness of the density profile and the velocity dispersion. We recalculate global profiles for the edge-on case, using the usual three density profiles, but now assume flat or declining rotation curves. To do this we modify the Catinella et al. (2006) template polyex curves by changing the $\alpha$ parameter as defined in Equation (3) and given in Table 1. This has the effect of changing the outer slope, but does not affect the inner rise of the rotation curve. For the flat rotation curves we simply set the value for the outer slope $\alpha$ to zero.

Choosing a value for $\alpha$ for the declining curves is more complicated, as we want to be guided by the declining curves observed in real galaxies, as opposed to modeling arbitrarily steeply declining slopes. We therefore fit the polyex model to the two most steeply declining $\mathrm{H}$ I rotation curves in the THINGS sample (de Blok et al. 2008), namely, NGC 4736 and NGC 2903. Unfortunately, for both these galaxies the inner slope is not tightly constrained (for NGC 4736 due to absence of $\mathrm{HI}$ in the center; for NGC 2903 due to streaming motions induced by the central bar), leading to some degeneracy between the $r_{\mathrm{PE}}$ and $\alpha$ fit parameters. For NGC 4736 we find values of $\alpha$ between $\sim-0.001$ and $\sim-0.002$. When the part of the rotation curve of NGC 2903 that is affected by the bar is not taken into account, we find $\alpha \sim-0.002$. Fitting the full curve yields $\alpha \sim-0.018$, though this value is likely affected by the uncertain inner rotation curve and the associated uncertainty in $r_{\mathrm{PE}}$. Nevertheless, to be conservative, we adopt a value $\alpha=-0.010$, which we regard as a hard limit on the value of $\alpha$ found in real disk 

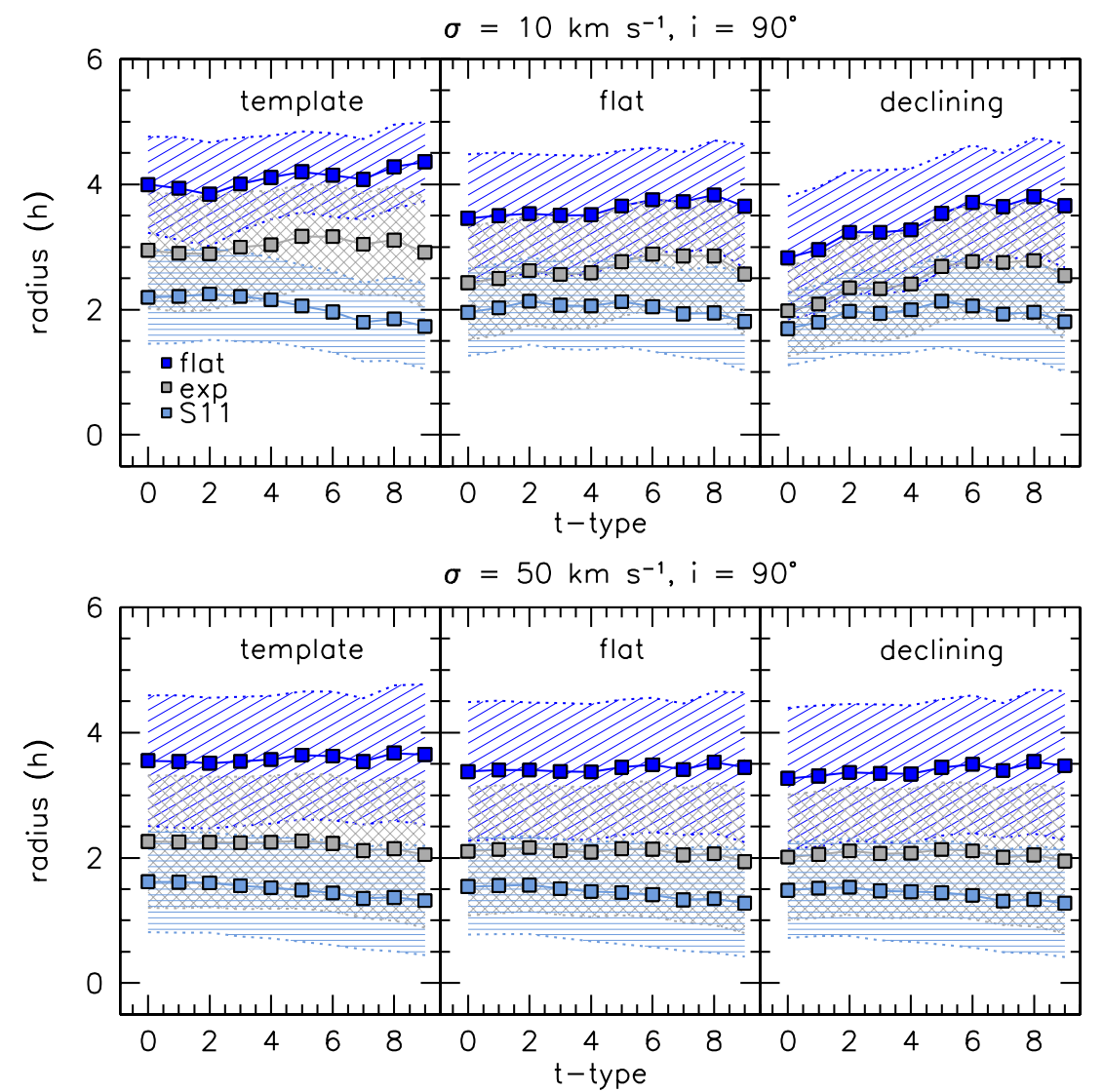

Figure 8. Top row: values of the characteristic radius at which $W_{50}$ occurs (as defined in Equation (4)) plotted as a function of $t$-type and assuming $\sigma=10 \mathrm{~km} \mathrm{~s}{ }^{-1}$. Shown are results for the flat density distribution model (dark blue), the exponential model (gray), and the S11 model (light blue). Hatched areas indicate the standard deviation in these radii as defined in Equation (5). The left panel shows the results for the Catinella et al. (2006) models used in the majority of this paper; the center and right panels shows the results for the respective models with a flat and a declining rotation curve, as discussed in the text. Bottom row: same, but now for $\sigma=50 \mathrm{~km} \mathrm{~s}^{-1}$.

(A color version of this figure is available in the online journal.)

galaxies with declining rotation curves. The results are shown in Figure 8.

We see that the characteristic radius of the flat density distribution has decreased somewhat for both flat and declining rotation curves, but they do not differ dramatically from the ones found for the original template rotation curves. Given the extreme outer slope we have adopted for the declining curve, it is therefore still justified to use a characteristic radius of $\sim 4 h$ to $\sim 5 h$ for determining the dynamical mass using any global profile that assumes a flat tracer profile.

The characteristic radii for the $\mathrm{S} 11$ distribution also show little change and remain around $2 h$. The results for $\sigma=50 \mathrm{~km} \mathrm{~s}^{-1}$ show much less difference when comparing the template, flat and declining rotation curve models, due to the more dominant velocity dispersion. Nevertheless, to first order the radii derived for the low-dispersion case also hold here. These results thus show that the shape of the global profile is not critically dependent on the shape of the rotation curve.

\section{SUMMARY}

The global velocity profile of a galaxy is often used as a means to estimate its dynamical mass. The profile is determined by the rotation curve of a galaxy, the gas distribution within a galaxy, and the velocity dispersion of the gas. We have investigated how the global profile changes as a function of these parameters. This is important due to the different ways global velocity profiles are typically determined at low and high redshifts. Local measurements of global velocity profiles mostly use $\mathrm{H} \mathrm{I}$ observations. Local H I disks usually have modest velocity dispersions, are extended with an almost constant surface density and sample the outer parts of the rotation curves well. On the other hand, CO (the main ISM tracer at high redshift) has a radial distribution that (at least for nearby galaxies) is known to be much steeper with an exponential decline. In addition, the velocity dispersion at high redshift is probably higher than in local galaxies. The difference in properties and distribution of the tracers can thus lead to differences in global profile widths and dynamical masses.

While the results presented here are generally applicable, for a comparison with real high-redshift galaxies we have to assume (1) that these galaxies are virialized and dominated by rotation, (2) that they are not majorly affected by interactions and/or asymmetries (though the latter will only have a small impact on $W_{50}$ ), (3) that rotation curves at high-redshift are to first order the same as at low redshift, and (4) that the range of gas density profiles modeled here overlaps with that in real galaxies. Choosing a different gas density profile from the range modeled here will account for the possibility that in high-redshift galaxies the gas distribution may be different from that in a low-redshift galaxy.

A first conclusion from the modeling of these profiles is that higher velocity dispersion and steeper density profiles result in less pronounced double-horned profiles. This is consistent 




Figure 9. Comparison of global profiles: Gray full profiles are from full three-dimensional TiRiFiC models, and the black dashed profile is from the analytical description. $t$ values refer to template curves. As the curves are practically indistinguishable we use the analytic curves as they are computationally much less expensive.

with high-redshift $\mathrm{CO}$ observations of galaxies, as discussed in Section 3.1. Galaxies with identical dynamics will have different global profiles, depending on the gas tracer used in the observations.

The steeper density profiles associated with $\mathrm{CO}$ give smaller velocity widths for otherwise identical rotation curves. The ratio between $\mathrm{CO}$ and $\mathrm{H}_{\mathrm{I}} W_{50}$ values changes from $\sim 0.95$ for the most luminous galaxies we modeled $\left(t=0\right.$, or $\left.M_{I} \sim-23.8\right)$ to $\sim 0.6$ for the faintest galaxies $\left(t=9\right.$ or $\left.M_{I} \sim-19.0\right)$.

This change affects the inferred slope of the TF-relation as well. The template rotation curves we use, in combination with a flat, constant-density distribution of the gas ("H I"), give a $W_{50}$-based TF slope of $\sim 3.7$. Using an S11 distribution for the gas ("CO") instead, decreases the slope to $~ 2.6$. These numbers assume a $10 \mathrm{~km} \mathrm{~s}^{-1}$ velocity dispersion, but the same decrease in slope is observed for the $50 \mathrm{~km} \mathrm{~s}^{-1}$ models.

When global profiles are used for dynamical mass estimates, resolved observations are usually not available and a radius must be assumed to calculate the dynamical mass. Usually an optical radius is used for this, though the definition of this radius not well constrained. To get around having to make an arbitrary choice for a radius, we calculate the effective radius of the rotation curve that gives rise to the measured $W_{50}$ value for a number of combinations of rotation curves and density profiles. We find that each gas density profile results in its own narrow range of characteristic radii, thus enabling a determination of the dynamical mass using a velocity width and a radius that is appropriate for the tracer used. Velocity widths $W_{50}$ measured in $\mathrm{H}$ I (with a flat density profile) typically originate at $\sim 5 h$, while velocity widths derived using the $\mathrm{S} 11$ density profile (equivalent to low-redshift $\mathrm{CO}$ distributions) originate at $\sim 2 h$. These results thus give us, for a range in gas density profile slopes, a consistent definition for the length scales to use in determining dynamical masses.

We thank Barbara Catinella for making available electronic versions of the template rotation curves. W.J.G.dB. was supported by the European Commission (grant FP7-PEOPLE2012-CIG 333939).

\section{APPENDIX A}

\section{COMPARISON WITH THREE-DIMENSIONAL MODELS}

The analytical method described in Obreschkow et al. (2009) is an efficient way to calculate a large number of global profiles. Here we compare some of these analytical global profiles with profiles derived from full three-dimensional models of rotating galaxies. Constructing the latter models is significantly more computationally intensive, but it enables us to derive global profiles using methods that are also used when analyzing real data. Good agreement between the two sets of profiles thus ensures that the conclusions we derive from the analytical models also apply to global profiles of real galaxies.

To construct the data cube models we use the TiRiFiC package (Józsa et al. 2007). TiRiFiC distributes cloud particles through a user-created three-dimensional (right ascension, declination, velocity) data cube, using user-defined geometric and kinematic parameters as distribution functions. Among the parameters that can be defined are the rotation curve, radially varying inclination and position angles, the surface density profile, the vertical distribution and extent of the gas and the velocity dispersion. TiRiFiC produces a data cube that can then be "observed" and treated like a real observation, and global profiles can be constructed using standard data analysis methods.

We have compared global profiles produced by both methods for a large range of template rotation curves, gas distributions, inclinations, and dispersions, and find the differences between the two methods to be negligible. As an example, we compare in Figure 9 the global profiles of three different edge-on galaxies, each with a different template rotation curve. A constant radial gas surface density was assumed for all three examples. We see very good agreement between the profiles from our threedimensional models and the analytic description. For the profiles shown in Figure 9, the mean absolute difference is $0.15 \%$; the maximum absolute difference is $0.4 \%$. These differences are purely due to specific choices made in the construction of the data cubes (e.g., pixel size and channel spacing) of the TiRiFiC models and are not systematic. For all practical purposes the profiles are identical and we proceed to use the analytical profiles derived using the Obreschkow et al. (2009) method.

\section{APPENDIX B}

\section{OTHER INCLINATIONS}

In the main text we have only discussed the case of edge-on galaxies. For these galaxies the signal of rotation is maximal and the effects of dispersion minimized. Here we explore how that balance changes for lower inclinations, and whether there is a significant impact on the measurements of velocity widths.

We investigate two additional cases: one where the inclination is $60^{\circ}$, the average inclination of a randomly oriented sample of galaxies, and one where the inclination is $30^{\circ}$, a practical lower limit on the inclination of real galaxies where reliable measurements of the rotation can still be done. For even lower inclinations the uncertainties in the associated inclination corrections become too large.

Figures 10 and 11 show the global profiles for the same galaxies as shown in Figure 1 , but now assuming $i=60^{\circ}$ 

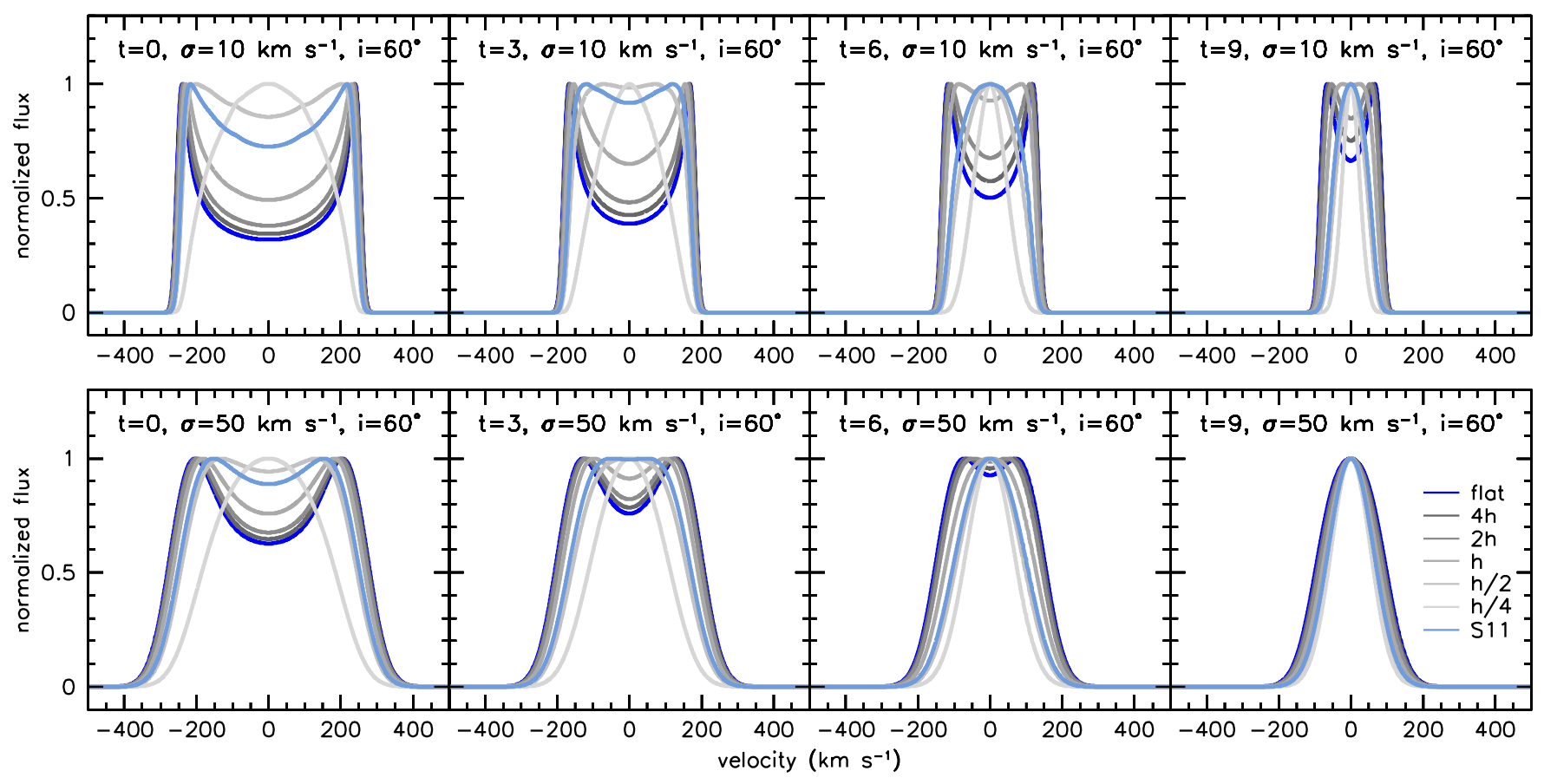

Figure 10. Same as Figure 2, but assuming $i=60^{\circ}$.

(A color version of this figure is available in the online journal.)


Figure 11. Same as Figure 2, but assuming $i=30^{\circ}$.

(A color version of this figure is available in the online journal.)

and $i=30^{\circ}$, respectively. It is clear that the effect of dispersion and profile steepness has become more pronounced. Especially for $i=30^{\circ}$ and $\sigma=50 \mathrm{~km} \mathrm{~s}^{-1}$ (bottom-row in Figure 11) we see that there is hardly any signature of rotation left, except for the most massive galaxies (lowest $t$-types). This is also seen in the changes in $W_{50}$ as a function of profile steepness as shown in Figures 12 and 13.

For $\sigma=10 \mathrm{~km} \mathrm{~s}^{-1}$ and $i=60^{\circ}$ there are only minor changes. Profile widths have shifted downwards due to the inclination, but in the steep density slopes part of the diagram they have more or less converged to the values found for the edge-on case, reflecting the very limited radial range that is probed in the latter case. Similar conclusions can be drawn for the $\sigma=50 \mathrm{~km} \mathrm{~s}^{-1}$ case.

For the $i=30^{\circ}$ case we see that for $\sigma=10 \mathrm{~km} \mathrm{~s}^{-1}$, apart from a much compressed range in $W_{50}$, the trends in velocity width with density profile steepness are similar as for $i=90^{\circ}$ and $i=60^{\circ}$. This situation is very different for the 


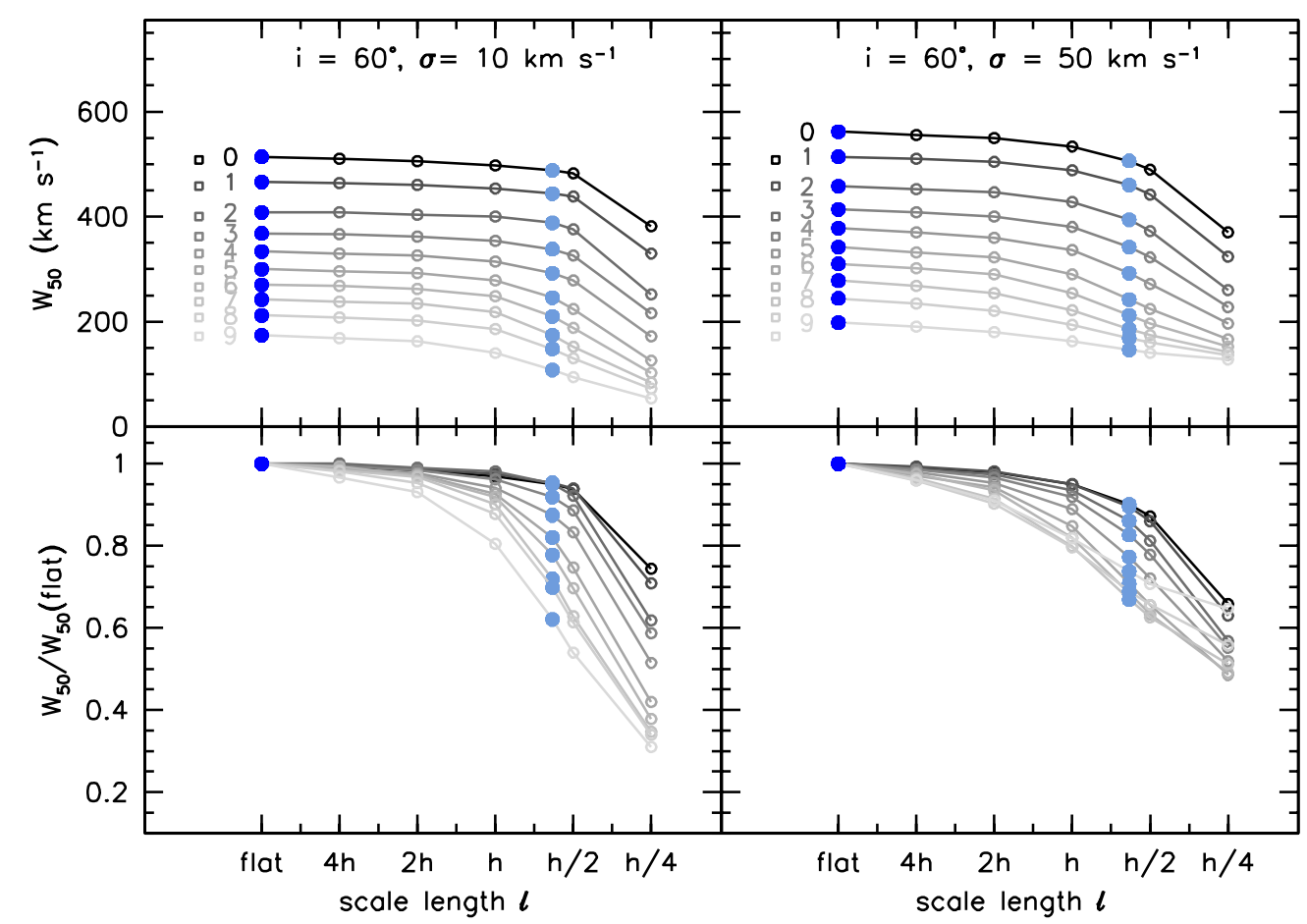

Figure 12. Same as Figure 3, but assuming $i=60^{\circ}$.

(A color version of this figure is available in the online journal.)

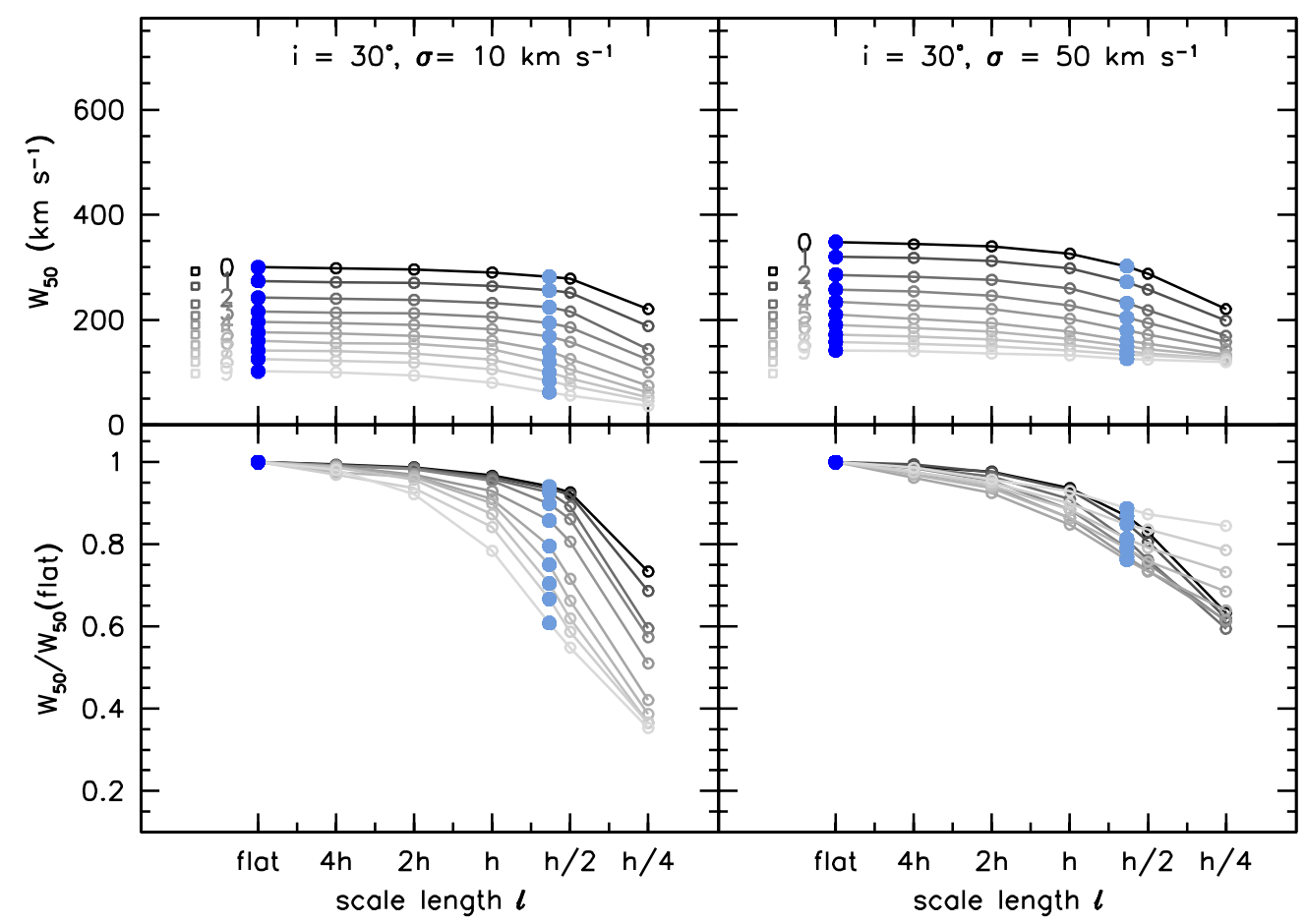

Figure 13. Same as Figure 3, but assuming $i=30^{\circ}$.

(A color version of this figure is available in the online journal.)

$50 \mathrm{~km} \mathrm{~s}^{-1}$ velocity dispersion. Here the large amplitude of the dispersion compared to the projected rotation velocity causes the velocity widths to be dominated by dispersion, especially for the steep density profiles and/or high $t$-type galaxies. This also means that the corresponding derived dynamical masses are not representative of the intrinsic masses.
Lastly we investigate the ratio of the velocity widths of profiles including a dispersion term and those with zero dispersion, as shown for edge-on galaxies in Figure 6, but here evaluated for $i=60^{\circ}$ and $i=30^{\circ}$. These are shown in Figure 14. It is clear that corrections become more dominant for lower inclination galaxies. 

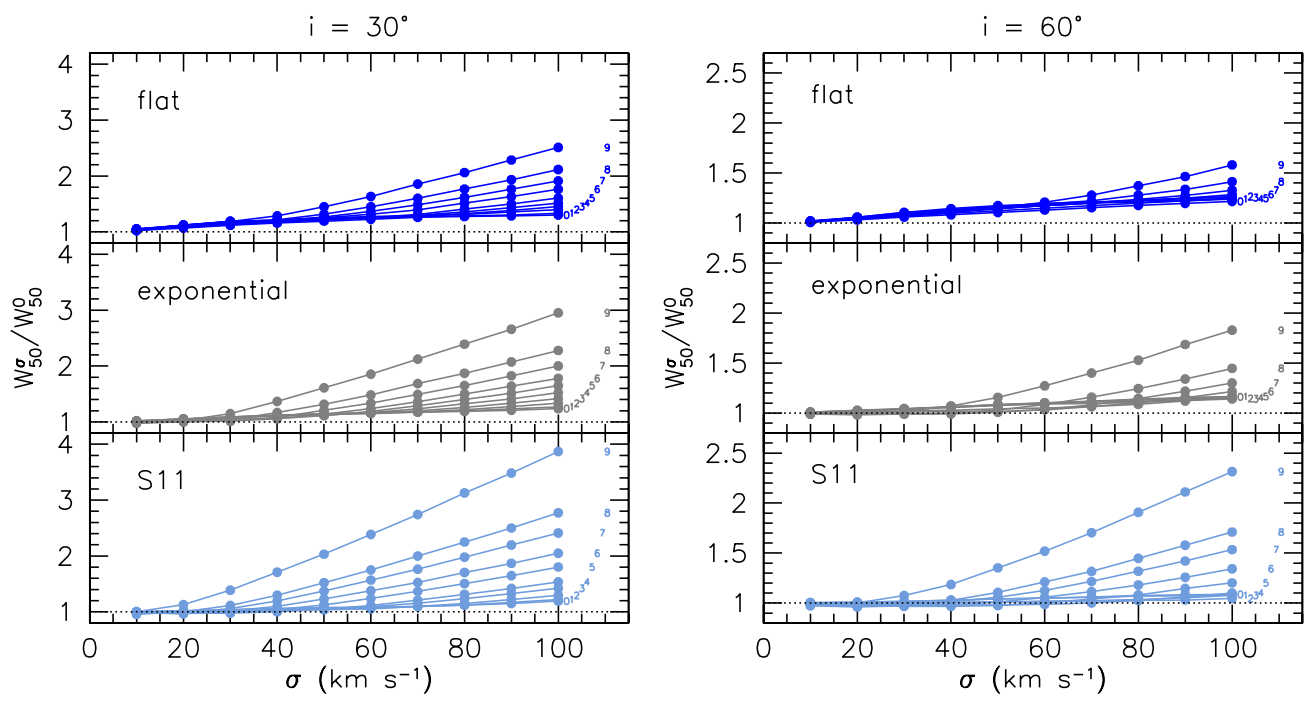

Figure 14. Ratio of the velocity widths that include velocity dispersion (as indicated on the horizontal axis) and the zero-dispersion velocity widths. The left panel shows corrections for $i=30^{\circ}$, the right panel for $i=60^{\circ}$. In each of the panels, from top to bottom are shown the ratios for the flat, exponential, and S11 density profiles. The correction curves are shown for each $t$-type, as indicated by the numbers in the right-most part of the plot.

(A color version of this figure is available in the online journal.)

\section{REFERENCES}

Abdalla, F. B., Blake, C., \& Rawlings, S. 2010, MNRAS, 401, 743 Bothwell, M. S., Smail, I., Chapman, S. C., et al. 2013, MNRAS, 429, 3047

Broeils, A. H., \& Rhee, M.-H. 1997, A\&A, 324, 877

Caldú-Primo, A., Schruba, A., Walter, F., et al. 2013, AJ, 146, 150

Carilli, C. L., \& Walter, F. 2013, AR\&AA, 51, 105

Catinella, B., Giovanelli, R., \& Haynes, M. P. 2006, ApJ, 640, 751

Daddi, E., Bournaud, F., Walter, F., et al. 2010, ApJ, 713, 686

de Blok, W. J. G., Walter, F., Brinks, E., et al. 2008, AJ, 136, 2648

de Jong, R. S. 1996a, A\&A, 313, 45

de Jong, R. S. 1996b, A\&A, 313, 377

Epinat, B., Tasca, L., Amram, P., et al. 2012, A\&A, 539, A92

Förster Schreiber, N. M., Genzel, R., Bouché, N., et al. 2009, ApJ, 706, 1364

Giovanelli, R., \& Haynes, M. P. 2002, ApJL, 571, L107

Gnerucci, A., Marconi, A., Cresci, G., et al. 2011, A\&A, 528, A88

Hodge, J. A., Carilli, C. L., Walter, F., et al. 2012, ApJ, 760, 11

Ianjamasimanana, R., de Blok, W. J. G., Walter, F., \& Heald, G. H. 2012, AJ, 144, 96

Józsa, G. I. G., Kenn, F., Klein, U., \& Oosterloo, T. A. 2007, A\&A, 468, 731

Lavezzi, T. E., \& Dickey, J. M. 1998, AJ, 116, 2672

Law, D. R., Steidel, C. C., Erb, D. K., et al. 2009, ApJ, 697, 2057
Leroy, A. K., Walter, F., Bigiel, F., et al. 2009, AJ, 137, 4670

Leroy, A. K., Walter, F., Brinks, E., et al. 2008, AJ, 136, 2782

Leroy, A. K., Walter, F., Sandstrom, K., et al. 2013, AJ, 146, 19

McGaugh, S. S. 2012, AJ, 143, 40

McGaugh, S. S., Schombert, J. M., Bothun, G. D., \& de Blok, W. J. G. 2000, ApJL, 533, L99

Miller, S. H., Sullivan, M., \& Ellis, R. S. 2013, ApJL, 762, L11

Newman, S. F., Genzel, R., Förster Schreiber, N. M., et al. 2013, ApJ, 767,104

Obreschkow, D., Croton, D., De Lucia, G., Khochfar, S., \& Rawlings, S. 2009, ApJ, 698, 1467

Riechers, D. A., Carilli, C. L., Maddalena, R. J., et al. 2011, ApJL, 739, L32

Schruba, A., Leroy, A. K., Walter, F., et al. 2011, AJ, 142, 37

Swinbank, A. M., Papadopoulos, P. P., Cox, P., et al. 2011, ApJ, 742, 11

Tacconi, L. J., Neri, R., Genzel, R., et al. 2013, ApJ, 768, 74

Tamburro, D., Rix, H.-W., Leroy, A. K., et al. 2009, AJ, 137, 4424

Tully, R. B., \& Fisher, J. R. 1977, A\&A, 54, 661

Tutui, Y., \& Sofue, Y. 1999, A\&A, 351, 467

Verheijen, M., van Gorkom, J. H., Szomoru, A., et al. 2007, ApJL, 668, L9

Verheijen, M. A. W. 2001, ApJ, 563, 694

Weiner, B. J., Willmer, C. N. A., Faber, S. M., et al. 2006, ApJ, 653, 1027 